\title{
Pulsed magnets with high field intensity for laser-plasma experiments and TDS spectroscopy
}

\author{
T. Krapivnitskaia, A. Luchinin, V. Malyshev, M. Morozkin, M. Starodubtsev, M. Proyavin, \\ A. Fokin, and M. Glyavin
}

Institute of Applied Physics, 46 Ulyanov Street, 603950 Nizhny Novgorod, Russia

The studies of different physical processes in presence of strong magnetic fields are one of the everlasting problems in experimental physics. Development of techniques and investigation of complex dynamics leads to the implementation of magnetic systems with fields more than $20 \mathrm{~T}$. The laser-plasma experiments [1] and time-domain spectroscopy [2] are one of the outstanding applications for strong magnetic fields. Conventional magnetic systems based on large superconducting magnets are not convenient for ultrafast spectroscopy due to remote access to the magnet coil and unavoidable dispersive elements. The solution is to use the small-scale pulsed systems, which provide easy alignment and strong field at the same time.

\section{Magnets for time-domain spectroscopy}

Terahertz time-domain spectroscopy (THz-TDS) is ideally suited for the study of coherent low-energy dynamics in condensed matter, determining the complex conductivity of material. The terahertz frequency range is rich in collective and elementary excitations in solids, many of which have magnetic origins such as cyclotron resonance, electron spin resonance, and magnon excitations. An external magnetic field provides a powerful tool for tuning these magnetic $\mathrm{THz}$ excitations through Landau and Zeeman quantization of electrons' orbital and spin energies. Probing these excitations through THz-TDS in high magnetic fields can provide valuable insight into the dynamics of interacting electrons. Therefore, there is a need for developing a source of strong magnetic field, suitable for TDS.

The IAP RAS has great experience in development and production of pulsed magnetic systems, which were mainly used for high-frequency gyrotrons [3]. The record magnetic field of $50 \mathrm{~T}$ in a bore of $9 \mathrm{~mm}$ (Fig. 1) was produced for the gyrotron with 1.3 $\mathrm{THz}$ frequency [4] along with a number of $30 \mathrm{~T}$ magnets with $2 \mathrm{~cm}$ bore. The developed technology is based on copper magnets encased in fiberglass body with liquid nitrogen as a coolant. The cooling to cryogenic temperatures reduces the resistance of copper, lowers the heating of the coil during the pulse and provides better pulse-to-pulse stability. Resulting coil operated with $1 \mathrm{~Hz}$ repetition rate and successfully produced more than 3000 pulses with field $>30 \mathrm{~T}$ and more than 100 pulses with field $>45 \mathrm{~T}$ without any degradation. The magnets of this type surely can find their application in experiments with time-domain spectroscopy in strong magnetic fields.

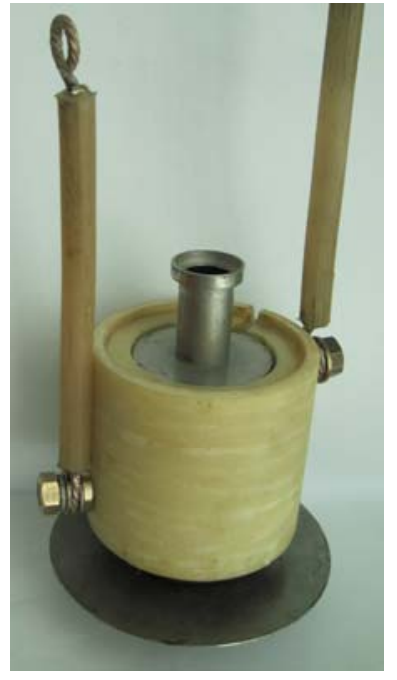

Fig. 1. The $50 \mathrm{~T}$ Pulsed magnet

\section{Magnets for laser-plasma experiments}

The studies on the formation of different plasma structures in strong magnetic field are of great interest for astrophysics and inertial fusion. Different astrophysical objects could be modeled in such downscaled laboratory experiments. Such astrophysical objects characterizes by the perturbation of the magnetic field structure and development of different magneto-hydrodynamic instabilities, which could not be studied analytically and should be studied through laboratory modeling [5].

The specially designed magnetic coil provides the needed conditions for investigation of plasma dynamics in magnetic field. The principal scheme of the setup and coil photo are presented in Fig. 2. To satisfy the requirements for the plasma formation and diagnostics the coil must have three mutually perpendicular apertures of free space inside the coil, that provide the possibility of positioning the target, irradiating the target with powerful nanosecond laser beam and observing the dynamics of plasma expansion perpendicular to the main axis of the direction of propagation of the plasma stream.

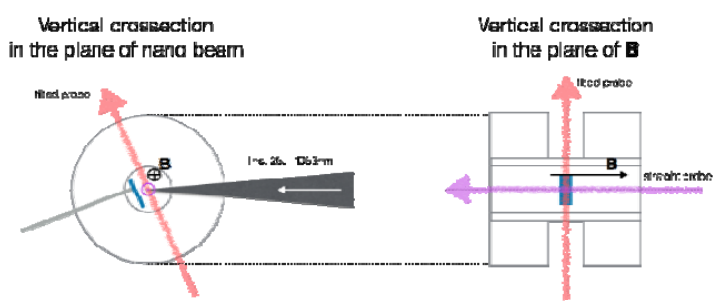

Fig. 2a. General view of the experimental setup. 


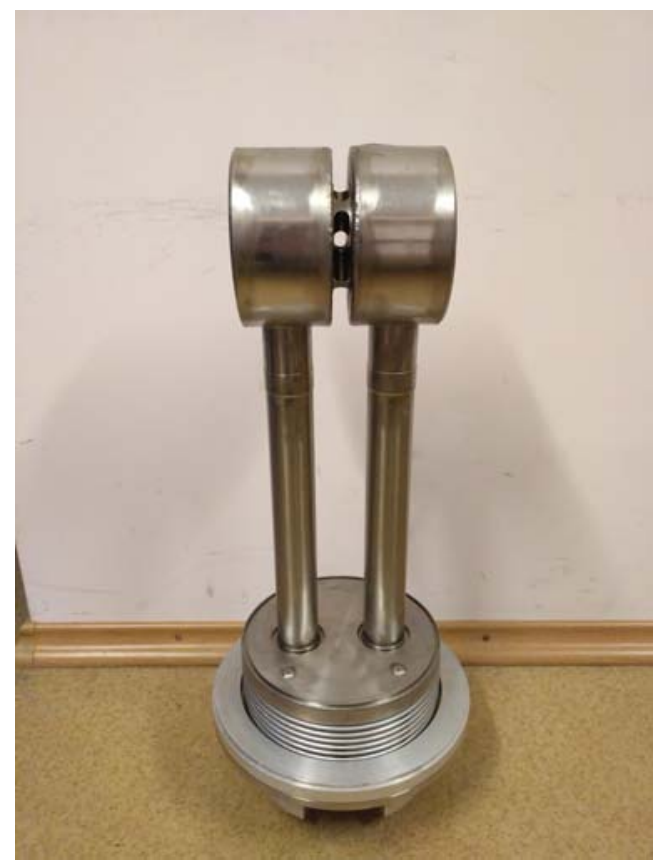

Fig. 2b. The pulsed magnet photo.

In order to satisfy the requirements, the Helmholtz magnetic coil was developed and manufactured by the IAP RAS. The coil current is controlled by a capacitor discharge unit with maximum current up to $8 \mathrm{kA}$, ensuring the maximum magnetic field of $15 \mathrm{~T}$. The discharge of the coil occurred over times of the order of several milliseconds. The field was considered stationary at time scales of the order of several microseconds, much larger than the times of the investigated plasma dynamics of the order of 100 ns. The magnetic field was practically uniform in the axial and radial directions at scales of the order of $20 \mathrm{~mm}$, which exceeded the characteristic scales of the investigating plasma ( $10 \mathrm{~mm})$. The repetition of the magnetic field amplitude and profile was ensured by cryogenic cooling of the system with liquid nitrogen. Typically, the coil can operate with repetition rate of 1 shot per 10 minutes. The construction of the magnet allowed switching of the direction of both coils, providing the co- and counter-directional setup of the magnetic field and opening up the possibility of investigation of plasma dynamics in different conditions. The measured profiles of magnetic field for both configurations are presented on Fig. 3. The magnet was already used in successful experiment, carried out on PEARL facility [6].

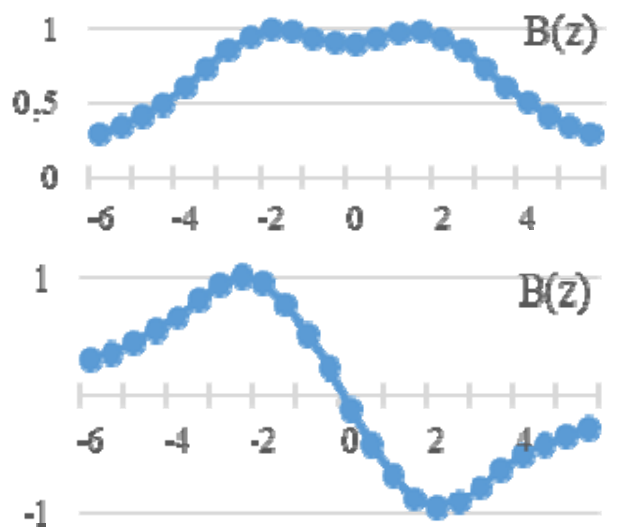

Fig. 3. Magnetic field profiles for co and counter-directional setup of coils.

\section{Conclusion}

The developed pulse magnets with fields up to 50 $\mathrm{T}$ and complex magnetic field structure open up the possibilities for investigation of properties of materials and laboratory modeling of astrophysical phenomena.

The work supported in part by the Russian Scientific Foundation under the project 14-12-00887.

\section{References}

1. Albertazzi, B. et al. Laboratory formation of a scaled protostellar jet by coaligned poloidal magnetic field // Science, 2014, V. 346, Vol. 346, No 6207, P. 325-328.

2. G. Timothy Noe et al. Single-shot terahertz timedomain spectroscopy in pulsed high magnetic fields // Optics Express, 2016, V. 24, No 26, P. 30328-30337.

3. M. Glyavin et al. A pulse magnetic field generator for terahertz gyrodevices // Instruments and Experimental Techniques, 2011, V. 54, No. 1, P. 77-80

4. Glyavin M.Y., Luchinin A.G., Golubiatnikov G.Y. Generation of 1.5-kW, 1-THz Coherent Radiation from a Gyrotron with a Pulsed Magnetic Field // Phys. Rev. Lett. 2008. V. 100, No 1. P. 15101

5. M. Starodubtsev et al. Laboratory investigation of magnetized laser plasmas expansion into the vacuum, ICONO/LAT 2016, Minsk, Belarus

6. Kurbatov, E.P. et al. Comparison of Dimensionless Parameters in Astrophysical MHD Systems and in Laboratory Experiments // Astronomy reports, 2018, V. 62, No. 8, P. 483-491 